\title{
Plantas medicinais x medicamentos anti-hipertensivos: interação medicamentosa
}

\author{
Medicinal plants $\mathbf{x}$ anti-hypertensive medications: drug interaction \\ Plantas medicinales $\mathbf{x}$ fármacos antihipertensivos: la interacción de drogas
}

Recebido: 17/11/2021 | Revisado: 24/11/2021 | Aceito: 25/11/2021 | Publicado: 09/12/2021

\author{
Jéssica Cristina Flores Porto \\ ORCID: https://orcid.org/0000-0003-0018-4332 \\ Universidade Salvador, Brasil \\ E-mail: jessicaccristina@outlook.com \\ Jheine Santana de Souza \\ ORCID: https://orcid.org/0000-0001-6443-9176 \\ Universidade Salvador, Brasil \\ E-mail: jheinesantana@hotmail.com \\ Luana Mascarenhas Brandão \\ ORCID: https://orcid.org/0000-0001-9225-7038 \\ Universidade Salvador, Brasil \\ E-mail: luana_mascarenhas@yahoo.com.br \\ Anny Carolinny Tigre Chaves \\ ORCID: https://orcid.org/0000-0002-9290-7368 \\ Universidade Salvador, Brasil \\ E-mail: annytigre@hotmail.com
}

\begin{abstract}
Resumo
O objetivo deste estudo é realizar uma revisão de literatura acerca das interações que podem ocorrer com o uso de plantas medicinais no contexto da hipertensão arterial. Trata-se de uma revisão narrativa de literatura, do tipo exploratória e de natureza qualitativa. Neste estudo, serão utilizados artigos pesquisados nas principais bases de dados na internet: Biblioteca Virtual em Saúde (BVS), Medical Literature Analysis and Retrieval System On-line (MEDLINE), Scientific Electronic Library Online (SciELO). As plantas medicinais apresentadas para tratar hipertensão foram Allium sativum, Alpinia zerumbet, Citrus sp., Passiflora sp., Cymbopogon citratus. O estudo revelou que entre as principais interações envolvidas na utilização de plantas medicinais associadas ao uso de fármacos para tratamento da hipertensão foram do tipo farmacodinâmicas, o que revela a necessidade de ter cautela na associação do uso de plantas aos medicamentos.
\end{abstract}

Palavras-chave: Plantas medicinais; Uso racional de plantas medicinas; HAS.

\begin{abstract}
The aim of this study is to carry out a literature review about the interactions that can occur with the use of medicinal plants in the context of arterial hypertension. This is a narrative literature review, exploratory and qualitative in nature. In this study, articles searched in the main databases on the internet will be used: Virtual Health Library (VHL), Online Medical Literature Analysis and Retrieval System (MEDLINE), Scientific Electronic Library Online (SciELO). The medicinal plants presented to treat hypertension were Allium sativum, Alpinia zerumbet, Citrus sp., Passiflora sp., Cymbopogon citratus. The study revealed that among the main interactions involved in the use of medicinal plants associated with the use of drugs for the treatment of hypertension were of the pharmacodynamic type, which reveals the need to be cautious in associating the use of plants with drugs.
\end{abstract}

Keywords: Medicinal plants; Rational use of medicinal plants; HAS.

\section{Resumen}

El objetivo de este estudio es realizar una revisión de la literatura sobre las interacciones que pueden ocurrir con el uso de plantas medicinales en el contexto de la hipertensión arterial. Se trata de una revisión de literatura narrativa, exploratoria y de naturaleza cualitativa. En este estudio se utilizarán los artículos buscados en las principales bases de datos en Internet: Biblioteca Virtual en Salud (BVS), Sistema de Análisis y Recuperación de Literatura Médica en Línea (MEDLINE), Biblioteca Electrónica Científica en Línea (SciELO). Las plantas medicinales presentadas para tratar la hipertensión fueron Allium sativum, Alpinia zerumbet, Citrus sp., Passiflora sp., Cymbopogon citratus. El estudio reveló que entre las principales interacciones involucradas en el uso de plantas medicinales asociadas al uso de fármacos para tratar la hipertensión fueron de tipo farmacodinámico, lo que revela la necesidad de ser cautelosos al asociar el uso de plantas con medicamentos.

Palabras clave: Plantas medicinales; Uso racional de plantas medicinales; HAS. 


\section{Introdução}

A Hipertensão arterial (HA) é condição clínica multifatorial caracterizada por elevação sustentada dos níveis pressóricos $\geq 140$ sistólica e/ou diastólica $90 \mathrm{mmHg}$. Frequentemente se associa a distúrbios metabólicos, alterações funcionais e/ou estruturais de órgãos-alvo, sendo agravada pela presença de outros fatores de risco, como dislipidemia, obesidade abdominal, intolerância à glicose e diabetes melito (DM). Mantém associação independente com eventos como morte súbita, acidente vascular encefálico (AVE), infarto agudo do miocárdio (IAM), insuficiência cardíaca (IC), doença arterial periférica (DAP) e doença renal crônica (DRC), fatal e não fatal (Costa et al.,2019).

Vale destacar, segundo Nogueira et al. (2014) que para o controle da HAS, além do tratamento medicamentoso, é necessário que o indivíduo abandone hábitos de vida que as deixe ainda mais vulneráveis a elevação de níveis pressóricos, como a ingesta em demasia de sódio, o sedentarismo, o tabagismo e o etilismo.

Em se tratando das terapias alternativas, Dias et al. (2016) destacam o uso de plantas medicinais, advertindo que a utilização das ervas medicinais concomitantemente com medicamentos pode ocasionar efeitos negativos, muitas vezes, justificadas pela falta de informação sobre as substâncias, além da mitificação de que produtos de origem vegetal não causam danos à saúde, podendo ser utilizados por qualquer pessoa.

É oportuno citar que a fitoterapia consiste em uma terapêutica que se caracteriza pela utilização de plantas medicinais. É importante lembrar que além da ação terapêutica comprovada de diversas plantas usadas popularmente, a fitoterapia faz parte da cultura de um povo, se configurando em um saber usado e disseminado pelas populações e preparados exclusivamente com plantas ou partes de plantas medicinais, que possuem propriedades reconhecidas de cura, prevenção, diagnóstico ou tratamento sintomático de doenças, validadas através de ensaios clínicos de fase 3 (Lucena \& Guedes,2020).

Segundo Campos et al. (2016), as plantas medicinais contêm princípios ativos que são produzidos durante o seu metabolismo, capazes de oferecer ação terapêutica. Os mesmos autores revelam que são classificadas como ervas medicinais, aquelas que possuem a capacidade de melhorar ou curar patologias, no entanto para afastar os riscos na utilização é necessário conhecer a planta.

No que diz respeito a nocividade das plantas medicinais para a saúde, Pontes et al. (2012) elenca que os problemas mais prevalentes são as intoxicações, as reações alérgicas e as interações medicamentosas. O autor destaca que a ocorrência desses efeitos pode ser ainda potencializada pelo equívoco em identificar as espécies consumidas, bem como a forma que as ervas são cultivadas, colhidas, estocadas, conservadas e produzidas.

As interações entre os medicamentos anti-hipertensivos e as substâncias existentes nas plantas medicinais podem ocorrer, o que colabora para a desconstrução de que as plantas não acarretam prejuízos à saúde, podendo ser utilizadas indiscriminadamente. As ervas podem interferir no processo de absorção, distribuição, metabolismo e excreção dos medicamentos anti-hipertensivos, antagonizando a ação do fármaco anti-hipertensivo, deste modo, reduzindo os efeitos esperados (Farias, 2016).

Diante disso, o objetivo deste estudo é realizar uma revisão de literatura acerca das interações que podem ocorrer com o uso de plantas medicinais no contexto da HAS.

\section{Metodologia}

Trata-se de uma revisão narrativa de literatura, do tipo exploratória e de natureza qualitativa. Neste estudo, foram utilizados artigos pesquisados nas principais bases de dados na internet: Biblioteca Virtual em Saúde (BVS), Medical Literature Analysis and Retrieval System On-line (MEDLINE) e Scientific Electronic Library Online (SciELO).

Conforme descreve Minayo (2010), o método qualitativo é adequado ao estudo de grupos delimitados, de histórias sociais, sob a perspectiva dos autores, e nas análises de discursos e de documentos. Tal abordagem se mostra mais do que 
adequada, necessária, para identificar quais os fatores colaboram para a ocorrência de interação entre plantas medicinais e medicamentos anti hipertensivos e quais são os tratamentos disponíveis.

Para realizar a busca foram utilizados os seguintes descritores: "Uso racional" e "plantas medicinais", e "Antihipertensivo" e seus correspondentes em inglês, "Rational use" and "medicinal plants", "Plants" and "antihypertensive".

Os artigos selecionados seguiram os seguintes critérios de inclusão: publicados entre 2006 e 2021, nos idiomas inglês e português, disponíveis gratuitamente para consulta. Os critérios de exclusão utilizados foram: artigos repetidos, aqueles que não atenderam a temática e os que não foram publicados durante o período delimitado.

\section{Resultados e Discussão}

\subsection{Hipertensão arterial: conceito, fatores predisponentes}

As VII Diretrizes Brasileiras de Hipertensão (2016) conceituam a HAS em um estado clínico multifatorial que pode ser identificada quando há um aumento sustentado da PA ( $\geq 140$ e/ou $90 \mathrm{mmHg}$ ). A HAS é normalmente associada a distúrbios do metabolismo, alterações nas funções e/ou estruturas dos órgãos alvo (Tabela 1).

Tabela 1 - Classificação da Pressão arterial sistêmica.

\begin{tabular}{ll}
\hline & \multicolumn{1}{c}{ VALORES DE REFERÊECIA (VR) } \\
\hline Normal & Pressão sistólica é $<120$ e a diastólica $<80 \mathrm{mmHg}$ \\
Pré-hipertensão & Pressão sistólica é $<121-139$ e a diastólica $<81-89 \mathrm{mmHg}$ \\
Hipertensão estágio 1 & Pressão sistólica entre $140-159$ e a diastólica entre $90-99 \mathrm{mmHg}$ \\
Hipertensão estágio 2 & Pressão sistólica entre $160-179$ e a diastólica entre $100-109 \mathrm{mmHg}$ \\
Hipertensão estágio 3 & Pressão sistólica $\geq 180$ e diastólica $\geq 110 \mathrm{mmHg}$ \\
\hline
\end{tabular}

Fonte: VII Diretrizes Brasileiras de Hipertensão (2016).

A doença pode apresentar maior gravidade quando o indivíduo apresenta outros fatores de risco, como por exemplo: dislipidemia, obesidade, Diabetes Mellitus (Mancia et al., 2013).

No que diz respeito aos fatores de risco para a HAS, Campos e Rodrigues Neto (2009) destacam o histórico familiar, situação em que se um dos pais forem portadores de hipertensão, os filhos estarão predispostos à $25 \%$ de serem acometidos pela HAS, sendo que estas chances aumentam em aproximadamente $60 \%$ quando o pai e a mãe são hipertensos.

Os mesmos autores indicam ainda como fator de risco para HAS, a dieta rica em sódio. Nesse sentido, a associação se dá porque o sódio favorece que o corpo faça um processo de retenção de líquido e essa retenção colabora para que ocorra uma elevação de carga sobre o coração.

Além dos fatores de risco já mencionados, a idade consiste em um fator bastante relevante, porque jovens e crianças, também podem ser hipertensas, porém, o diagnóstico em grande parte dos casos só é realizado a partir dos 35 anos. Vale destacar que aos 50 anos, aproximadamente metade da população já é hipertensa, onde pode-se aumentar a medida em que a idade for aumentando (Ribas \& Silva, 2014).

Segundo Nascimento; Gomes e Sardinha (2011), o sedentarismo é um fator de risco para a HAS, visto que o risco se eleva quando o indivíduo passa bastante tempo sentado no trabalho, em casa e se não tiver uma adesão a prática de exercícios físicos regularmente.

O etilismo também foi apontado como um fator de risco para a elevação da pressão arterial, já que o consumo regular e de maneira abusiva de bebida alcoólica colabora para que a pressão arterial se eleve, ocasionando insuficiência cardíaca, batimentos irregulares e até mesmo acidente vascular cerebral (Nobre et al., 2013). 
Conforme Nogueira (2014), o tratamento da hipertensão arterial exige além do uso de fármacos, transformação nos hábitos rotineiros de vida, sendo necessário que os profissionais da saúde promovam estratégias com vistas a controlar a hipertensão arterial, definição do diagnóstico clínico e na decisão de propor uma terapia, na educação da pessoa com HAS acerca de sua morbidade e da importância do paciente hipertenso em aderir ao tratamento.

Para tanto, o mesmo autor explica que o paciente tem que se sentir encorajado e motivado para que este não abandone o tratamento, já que grande parte da população que apresenta hipertensão traz consigo outras comorbidades, como diabetes, dislipidemia e obesidade, acarretando em consequências relevantes no que diz respeito ao gerenciamento das ações terapêuticas necessárias, exigindo, motivação e educação permanente.

\subsection{Tratamento da hipertesão arterial sistêmica}

No tratamento da hipertensão arterial sistêmica, estão inclusos o uso de fármacos e também medidas não medicamentosas, com vistas a redução da pressão arterial, proteção de órgãos alvo, prevenção de eventos cardiovasculares e renais (Freitas et al., 2015).

\subsubsection{Tratamento não medicamentoso}

O tratamento não medicamentoso tem sido uma alternativa bastante eficaz na redução da pressão arterial, embora sejam limitados devido a perda de adesão a médio e longo prazo (Oliveira et al., 2013).

No tratamento não medicamentoso estão envolvidos o controle do peso, medidas nutricionais, prática de exercícios físicos, abandono do tabagismo e do etilismo. Entre os benefícios da terapia não medicamentosa estão o baixo custo e risco mínimo; controle da pressão arterial, controle de outros fatores de risco; aumento da eficácia do tratamento medicamentoso; redução do risco cardiovascular (Mascarenhas, 2016; Lee et al.,2016).

Machado et al. (2016), avaliaram a adesão ao tratamento não farmacológico da hipertensão arterial sistêmica por meio de parâmetros antropométricos, bioquímicos, clínicos e dietéticos antes e após três estratégias de educação em saúde e nutrição. A amostra foi constituída de 212 indivíduos com diagnóstico de hipertensão arterial sistêmica que atenderam aos critérios de inclusão/exclusão. Os participantes foram alocados em três grupos de forma a avaliar o impacto das modalidades de intervenção realizadas mensalmente durante 12 meses. Como achado mais relevante, o estudo apontou que no grupo 1 houve redução estatisticamente significante da circunferência da cintura. Nos grupos 2 e 3, os resultados foram estatisticamente significantes para o peso, índice de massa corporal e circunferência da cintura. Foram verificadas reduções estatisticamente significantes na glicemia, no colesterol total e lipoproteína de baixa densidade nos grupos 1 e 2 . As intervenções também foram capazes de promover alterações favoráveis no consumo médio per capita de óleo, açúcar e sal em todos os grupos.

Além disso, o estudo de Machado et al. (2016), evidenciou que as intervenções educativas propiciaram resultados positivos sobre a adesão ao tratamento não farmacológico da HAS, considerando os parâmetros antropométricos (peso, índice de massa corporal e circunferência da cintura), bioquímicos (glicemia, colesterol total e lipoproteína de baixa densidade), e dietéticos (consumo médio per capita de óleo, açúcar e sal).

Almeida et al. (2019), realizaram um estudo com a finalidade de relatar um caso de um idoso portador de HAS, frequentador de uma casa de apoio a idosos no município de Quixadá, Ceará, promovendo o conhecimento sobre a terapia não medicamentosa e incentivando o idoso a aderi-la, evidenciando seus benefícios, além de classificar os tipos de terapia e como ela pode contribuir para uma melhor qualidade de vida.

Assim como Weber et al. (2014), Almeida et al. (2019), mostraram que o acesso às informações sobre os medicamentos utilizados e a intervenção de ações educativas minimizam a ocorrência de reações adversas aos medicamentos 
usados e favorecem a qualidade de vida do paciente, tornando a farmacoterapia eficaz, além de conscientizá-lo quanto ao risco do fumo no organismo e fazê-lo liminar essa prática. O paciente deixou de fumar e, no final do período do estudo, os dados coletados mostraram redução do peso e dos valores da PA.

\subsubsection{Tratamento medicamentoso}

Quanto ao tratamento medicamentoso, Freitas et al. (2015) apontam que a decisão pela terapêutica dele levar em conta os valores pressóricos, a existência ou não de lesão em órgãos-alvo e os fatores de risco associados, que permitem estratificar o risco do paciente a ser tratado. A redução da pressão arterial com medicamentos tem a capacidade de diminuir a morbidade e mortalidade por doenças de etiologia cardiovascular em pessoas acometidas por HAS.

Desde que seja indicado o tratamento com medicamentos, o paciente dever ser orientado sobre a necessidade da administração continua, do ajuste eventual de doses, da troca ou associação de drogas e sobre os possíveis efeitos adversos (Magnabosco et al., 2015).

É importante destacar que a administração em baixas doses, de acordo com Serrate Mengue e colaboradores (2016), reduz os riscos da ocorrência de efeitos adversos, contudo não interfere na eficácia do mecanismo de ação, sobretudo, quando são associados a outras classes.

\subsubsection{Medicamentos Diuréticos}

O mecanismo de ação dos diuréticos está associado aos efeitos natriuréticos (eliminação do sódio pelos rins), além da redução do volume extracelular e a partir da quarta semana já se pode observar que o volume circulante já se encontra normalizado provocando uma diminuição na resistência vascular periférica (Simão et al., 2016).

Barroso et al. (2020) explicam que se deve dar preferência aos Diuréticos (DIU) tiazídicos (hidroclorotiazida) ou similares (clortalidona e indapamida) em doses baixas, pois são mais suaves e com maior tempo de ação, reservando-se os DIU de alça (furosemida e bumetanida) às condições clinicas com retenção de sódio e água, como a insuficiência renal (creatinina > $2,0 \mathrm{mg} / \mathrm{dL}$ ou o ritmo de filtração glomerular estimado $\leq 30 \mathrm{~mL} / \mathrm{min} / 1,73 \mathrm{~m} 2$ ) e situações de edema (IC, síndrome nefrítica). Os DIU poupadores de potássio (espironolactona e amilorida) costumam ser utilizados em associação aos tiazídicos ou DIU de alça.

Neto et al. (2017) destaca ainda que a espironolactona tem sido habitualmente utilizada como o quarto medicamento a ser associado aos pacientes com HAS resistente e refratária. Na lista dos efeitos adversos associados ao uso dos diuréticos estão: fraqueza, câimbras, hipovolemia e disfunção erétil, hipopotassemia, arritmias ventriculares, bem como a intolerância à glicose, pois reduzem a liberação de insulina, com isso o hipertenso pode desenvolver Diabetes tipo II, devido ao aumento de glicemia (Silva et al., 2015).

Vale destacar que os Diuréticos não devem ser usados concomitantemente com digitálicos, pois podem provocar hipopotassemia, nem com os anti-inflamatórios não esteroidais, porque provoca antagonismo do efeito diurético. Quando administrado com lítio, ocasiona aumento dos níveis séricos do lítio e com inibidores de convertase e suplemento de potássio pode provocar Hiperpotassemia. Além disso, a espironolactona pode causar ginecomastia e hiperpotassemia, sendo este distúrbio eletrolítico mais frequente em pacientes com déficit de função renal. Há relatos de que a indapamida pode ter um melhor perfil metabólico em comparação com a hidroclorotiazida (Porto, 2015).

\subsubsection{Medicamentos Agentes de ação central}

Os agentes de ação central agem no sentido de reduzir o débito cardíaco e a secreção de renina, readaptando os barorreceptores e diminuição das catecolaminas nas sinapses nervosas. Vale destacar que o uso destes fármacos é restrito as 
pessoas portadoras de doença pulmonar obstrutiva crônica, nos bloqueios atrioventriculares de $2^{\circ}$ e $3^{\circ}$ graus e devem ser utilizados com prudência na população acometidas pelas vasculopatias periféricas. São representantes desse grupo: metildopa, clonidina e o inibidor dos receptores imidazolínicos rilmenidina (Issa et al., 2021).

Essa classe apresenta como reações adversas: febre, anemia hemolítica, galactorreia e disfunção hepática, sonolência, sedação, boca seca, fadiga, hipotensão postural e disfunção erétil. Uma das causas para não adesão a essa classe está na disfunção erétil. Além disso, a metildopa pode provocar reações autoimunes, como febre, anemia hemolítica, galactorreia e disfunção hepática, que, na maioria dos casos, desaparecem com a interrupção do uso (Cupertino et al., 2016).

No desenvolvimento de uma reação adversa, a metildopa pode ser substituída por outro alfa-agonista central. A clonidina apresenta risco do efeito rebote com a descontinuação, especialmente quando associada aos betabloqueadores, e pode ser perigosa em situações pré-operatórias. A retirada gradual em duas a quatro semanas evita o efeito rebote. Os medicamentos dessa classe apresentam reações adversas decorrentes da ação central, como sonolência, sedação, boca seca, fadiga, hipotensão postural e disfunção erétil (Oigman et al., 2015).

\subsubsection{Medicamentos Betabloqueadores}

Os betabloqueadores atuam na diminuição do débito cardíaco e da secreção de renina (Chequer et al., 2016). No que diz respeito às reações adversas, Peres e Castro (2015) apontam as principais reações como o broncoespasmo, bradicardia, vasoconstrição periférica, insônia, astenia, disfunção sexual, Intolerância à glicose; elevação do LDL e redução do HDL.

Os betabloqueadores podem ser diferenciados em três categorias, de acordo com a seletividade para ligação aos receptores adrenérgicos: 1) não seletivos - bloqueiam tanto os receptores adrenérgicos beta-1, encontrados principalmente no miocárdio, quanto os beta-2, encontrados no músculo liso, nos pulmões, nos vasos sanguíneos e em outros órgãos (propranolol, nadolol e pindolol, este último apresentando atividade simpatomimética intrínseca, agindo como um agonista adrenérgico parcial e produzindo menos bradicardia); 2) cardiosseletivos - bloqueiam preferencialmente os receptores beta-1 adrenérgicos (atenolol, metoprolol, bisoprolol e nebivolol, que é o mais cardiosseletivo); e 3) com ação vasodilatadora - manifesta-se por antagonismo ao receptor alfa-1 periférico (carvedilol) e por produção de óxido nítrico (nebivolol) (Carvalho et al, 2016).

Os Betabloqueadores (BB) são contraindicados em pacientes com asma, doença pulmonar obstrutiva crônica e bloqueio atrioventricular de segundo e terceiro graus. Além disso, podem acarretar intolerância à glicose, induzir ao aparecimento de novos casos de diabetes melito, hipertrigliceridemia, elevação do colesterol-LDL e redução do colesterolHDL. O impacto sobre o metabolismo da glicose é potencializado quando são utilizados em combinação com DIU. Os BB de terceira geração (carvedilol e nebivolol) têm impacto neutro ou até podem melhorar o metabolismo da glicose e lipídico, possivelmente pelo efeito vasodilatador, com diminuição da resistência à insulina e melhora da captação de glicose pelos tecidos periféricos (Issa et al.,2021).

\subsubsection{Medicamentos Alfa bloqueadores}

Os alfas bloqueadores são drogas com ação antagonista competitiva dos alfa-receptores pós-sinápticos, desta maneira reduzem a resistência vascular periférica, sem interferir no débito cardíaco (Cupertino et al., 2016). No que se refere às reações provocadas pelo uso dos alfas bloqueadores estão a hipotensão sintomática na primeira dose e incontinência urinária em pessoas do sexo feminino. Representantes dessa classe utilizados como medicamentos anti-hipertensivos são a doxazosina e a prazosina (Cruz et al., 2015).

Os Alfa bloqueadores podem provocar hipotensão sintomática na primeira dose. O fenômeno de tolerância é frequente, necessitando aumento da dose para manter o efeito anti-hipertensivo (taquifilaxia). A incontinência urinária em 
mulheres pode ser causada pelo uso de alfa bloqueadores. Há evidência de que os pacientes tratados com doxazosina têm maior risco de incidência de insuficiência cardíaca (Feitosa et al.,2020).

\subsubsection{Medicamentos bloqueadores dos canais de cálcio}

Os bloqueadores dos canais de cálcio (BCC) agem de modo a reduzir a resistência vascular periférica por diminuição da concentração de cálcio nas células musculares lisas. Dentre as reações mais comuns da utilização deste medicamento estão a cefaleia, tontura, rubor facial e edema de extremidades, hipertrofia gengival e prisão de ventre (Elliott, 2011).

OS BCC são classificados em dois tipos básicos: os di-hidropiridínicos e os não di-hidropiridínicos. Os dihidropiridínicos (anlodipino, nifedipino, felodipino, manidipino, levanlodipino, lercanidipino, lacidipino) exercem efeito vasodilatador predominante, com mínima interferência na Frequência cardíaca e na função sistólica, sendo, por isso, mais frequentemente usados como medicamentos anti-hipertensivos. Os BCC não di-hidropiridínicos, como as difenilalquilaminas (verapamila) e as benzotiazepinas (diltiazem) têm menor efeito vasodilatador e agem na musculatura e no sistema de condução cardíacos. Por isso, reduzem a frequência cardíaca, exercem efeitos antiarrítmicos e podem deprimir a função sistólica, principalmente nos pacientes que já tenham disfunção miocárdica, devendo ser evitados nessa condição (Yugar-Toledo et al.,2020).

No tocante aos efeitos adversos associados aos bloqueadores dos canais de cálcio, o edema maleolar costuma ser o efeito colateral mais registrado e resulta da própria ação vasodilatadora (mais arterial que venosa), promovendo a transudação capilar. A cefaleia latejante e as tonturas também são comuns. O rubor facial é mais comum com os BCC di-hidropiridínicos de ação rápida. A hipercromia do terço distal das pernas (dermatite ocre) e a hipertrofia gengival são efeitos adversos ocasionais (Suchard et al.,2020).

Os efeitos adversos costumam ser dose-dependentes, podem causar intolerância aos BCC di-hidropiridínicos e, às vezes, resultam em resistência ao tratamento. Nesses casos, pode ser testada a utilização de BCC lipofílicos (manidipino, lercanidipino, lacidipino) ou o levanlodipino em baixas doses. A verapamila e o diltiazem podem agravar a IC, além de causar bradicardia e bloqueio atrioventricular. Observa-se a obstipação intestinal com a verapamila (Malachias et al., 2016).

\subsubsection{Medicamentos Inibidores da enzima conversora da angiotensina}

Adicionalmente, os Inibidores da enzima conversora da angiotensina (ECA) devem ser prescritos na ocorrência de hipertensão arterial associada ao diabetes e à insuficiência cardíaca. Esta classe de medicamentos minimiza a morbidade e mortalidade cardiovasculares em pessoas com disfunção sistólica do ventrículo esquerdo, sintomáticos ou não, atuando principalmente no processo de remodelação ventricular. São indicados também para prevenção da progressão da insuficiência renal, sobretudo, a pacientes que apresentam diabetes. Os principais representantes dessa classe são o ramipril, captopril, enalopril, fosinopril, lisinopril e quinapril (Vejakama et al., 2012).

Contudo, as principais reações adversas associadas ao uso dos Inibidores da ECA estão a tosse seca, alteração do paladar, erupção cutânea e edema angioneurótico, hiperpotassemia e aumento da ureia e creatinina na insuficiência renal (Andrade, 2015). Entre as reações adversas, a tosse seca está entre as principais reações para a mudança do medicamento.

\subsubsection{Medicamentos Bloqueadores dos receptores da AT1 e 2}

Os bloqueadores dos receptores da AT1 e 2 (BRA) possuem como mecanismo de ação o bloqueio especifico dos receptores AT1 da Angiotensina 2, desta forma, exercem efeitos cardioprotetores e nefro protetores em diabéticos tipo II com nefropatia estabelecida. Essa classe não apresenta reações adversas significativas. Sendo assim, seu modo especifico de ação, 
minimiza os seus efeitos adversos. São representantes dessa classe a Losartana, Candesartana, Eprosartana, Ibersatana, Telmisartan, Valsartana (Reis Filho, 2015).

De forma semelhante aos IECA, os BRA podem promover a redução inicial da filtração glomerular por vasodilatação das arteríolas eferentes, diminuindo a pressão de filtração glomerular, mas esse efeito é nefro protetor a longo prazo. Pelas mesmas razões dos IECA, podem causar hipercalemia, especialmente na presença de insuficiência renal, e são contraindicados na gravidez, devendo os mesmos cuidados serem tomados em mulheres em idade fértil (Malachias et al.,2016).

\subsubsection{Medicamentos Vasodilatadores diretos}

Existem ainda os medicamentos que atuam na musculatura da parede vascular, os vasodilatadores diretos, os quais agem promovendo relaxamento muscular com consequente vasodilatação e redução da resistência vascular. Os principais medicamentos vasodilatadores de ação direta são: Hidralazina e Minoxidil (Vongpatanasin et al., 2011).

Este medicamento apresenta como reação adversa retenção hídrica e taquicardia reflexa, deste modo não devem ser prescritos como monoterapia, devendo ser associados a diuréticos ou betabloqueadores. Os efeitos colaterais incluem cefaleia, flushing, taquicardia reflexa e reação lupus-like (dose-dependente). Seu uso pode também acarretar anorexia, náusea, vômito e diarreia. Os vasodilatadores podem provocar retenção de sódio e água, com o aumento do volume circulante e da taquicardia reflexa (Monteiro, 2017).

É oportuno destacar que a terapêutica provoca grande influência na adesão ao tratamento, sobretudo, no que diz respeito aos efeitos adversos a complexidade dos esquemas terapêuticos. Aonde esses efeitos consistem em fatores relevantes para que o paciente faça aderência ao tratamento, embora, se tenha no mercado drogas mais modernas e com efeitos colaterais mínimos, os custos e a deficiência no acesso a estes medicamentos fazem com que a adesão seja baixa (Oigman et al., 2015).

\subsection{As Plantas Medicinais}

As ervas medicinais dizem respeito as mais remotas alternativas utilizadas para tratar doenças de todas as naturezas, ou seja, o uso destas para prevenir ou parar curar patologias é um costume que que sempre existiu na história da humanidade. O marco sobre a utilização de plantas medicinais é registrado 500 a. C., contudo, os primeiros registros fitoterápicos são datados no período 2.838-2.698 a. C., quando o imperador chinês Shen Nung catalogou 365 ervas medicinais (Pereira et al.,2015).

Planta medicinal consiste em toda espécie vegetal que ofereça um benefício de natureza curativa para determinada comunidade, ou seja, que possui uma propriedade real ou imaginária, utilizada pela comunidade para um ou mais objetivos específicos de cura, que seja utilizada para prevenir, tratar e curar problemas, distúrbios ou patologias de pessoas e animais (Carvalho et al.,2015).

As plantas medicinais acarretam exposição a riscos relevantes na saúde de quem as utiliza de forma inadequada, o principal risco, são as interações medicamentosas que podem ocorrer entre medicamentos sintéticos e fitoterápicos (Almeida; Barbosa \& Santana,2016).

\subsubsection{Plantas medicinais e interações com medicamentos anti-hipertensivos}

As plantas medicinais que apresentaram interações com medicamentos anti-hipertensivos mais evidenciadas neste levantamento foram: Alliaceae Allium sativum L., Alpinia zerumbet, Passiflora sp, Rutaceae Citrus sp, Verbenaceae Lippia alba (Mill.), Poaceae Cymbopogon citratus (DC.) Stapf.. No Quadro 1, estão demonstradas as plantas que mais apareceram citadas nas bases de dados, bem com nome científico, nome popular, parte utilizada e os efeitos. 
Tabela 2 - Plantas medicinais que acarretam interações quando utilizadas em associação com anti-hipertensivos.

\begin{tabular}{cccc}
\hline NOME CIENTÍFICO & NOME POPULAR & PARTE UTILIZADA & EFEITOS \\
\hline $\begin{array}{c}\text { Alliaceae Allium sativum } \\
\text { L. }\end{array}$ & Alho & Folha & $\begin{array}{c}\text { Coadjuvante no } \\
\text { tratamento e hipertensão } \\
\text { arterial }\end{array}$ \\
\hline $\begin{array}{c}\text { Alpinia } \text { zerumbet } \\
\text { Passiflora } \text { sp. }\end{array}$ & Cardamomo & Folha & $\begin{array}{c}\text { Redução da pressão } \\
\text { arterial }\end{array}$ \\
\hline $\begin{array}{c}\text { Rutaceae } \text { Citrus } \text { sp. } \\
\text { Verbenaceae }\end{array}$ & Maracujá & Folha & $\begin{array}{c}\text { Redução da pressão } \\
\text { arterial }\end{array}$ \\
\hline Lippia alba $($ Mill. $)$ & Laranja & Folha, fruto & $\begin{array}{c}\text { Redução da pressão } \\
\text { arterial }\end{array}$ \\
\hline $\begin{array}{c}\text { Cymbopogon } \text { citratus } \\
\text { (DC.) } \text { Stapf },\end{array}$ & Erva-cidreira & Folha,flor & $\begin{array}{c}\text { Redução da pressão } \\
\text { arterial }\end{array}$ \\
\hline
\end{tabular}

Fonte: Dados da pesquisa (2021).

Farias (2016) e Carvalho et al. (2021) explicam que o potencial de interação planta/medicamento tem dependência da presença e mecanismo de ação de alguns metabólitos secundários e que os medicamentos anti-hipertensivos podem ter seus efeitos antagonizados quando usados com plantas medicinais com atividade hipertensiva, vasoconstritora e de retenção de líquido, e potencializados quando utilizados com plantas com atividade hipotensiva, vasodilatadora e diurética.

\section{Allium sativum $L$.}

O alho é constituído por compostos sulfurados, aliina, alicina e ajoeno, além de glicosídeos, monoterpenoides, enzimas, vitaminas, minerais e flavonóides (canferol e quercetina), saponinas (Lima et al.,2020).

No que diz respeito ao mecanismo de ação, Souza et al. (2017), Lima et al. (2018), Lima et al. (2020), destacaram que os compostos sulfurados apresentam atividade vasodilatadora in vitro mediada por liberação de óxido nítrico e que a utilização de alho pode ser capaz de reduzir a expressão das isoformas CYP3A4, CYP3A5, CYP3A7, CYP2C9, CYP2C19 e CYP2E1 e da glicoproteína-P e pode aumentar a expressão da CYP2C9, CYP3A1, CYP1A1.

Quanto as interações medicamentosas, Carneiro e Comarella (2016), Dias et al. (2017), Souza et al. (2017) estão em concordância e referiram em seus estudos que podem ocorrer interações sinérgicas quando o alho é utilizado concomitantemente com anti-hipertensivo da classe $\beta$-bloqueador, que age reduzindo o débito cardíaco e que reduz a secreção de renina, causa readaptação de barorreceptores e redução das catecolaminas nas sinapses nervosas, esse medicamento atua ainda proporcionando vasodilatação pelo aumento da síntese e liberação endotelial de óxido nítrico .

Lima et al. (2020) acrescenta que existe um efeito similar ao dos compostos sulfurados presentes no alho, ocorrendo uma potencialização do efeito do medicamento e as interações com os anti-hipertensivos inibidores da enzima conversora da angiotensina (ECA), provoca um aumento do efeito hipotensor do medicamento e os medicamentos da classe dos antagonistas dos canais de cálcio sofrem metabolização no CYP450, sendo o verapamil metabolizado principalmente pela isoforma CYP3A4, os $\beta$ - bloqueadores (propranolol) são metabolizados pela isoforma CYP2D6 que sofrem interferência na presença do alho sugerindo ocorrendo interações farmacocinéticas quando utilizados em concomitância com o alho.

\section{Alpinia zerumbet}

Souza et al. (2017) mostraram que o Cardamomo é composto por mono e sesquiterpenos, com maior concentração de terpinen-4-ol e 1,8-cineol, compostos com ação hipotensora e vasodilatadora, no entanto, entre os componentes fixos, os mais importantes são os flavonóides e as kava-pironas e a presença de flavonóides contribui com ação anti-hipertensiva da planta. 
Castro et al. (2016), Cunha et al. (2016), Lins et al. (2020) discutiram sobre o mecanismo de ação e evidenciaram que o tratamento com óleo essencial de Alpinia zerumbet reduz a pressão arterial devido a presença dos componentes terpinen-4-ol e 1,8-cineol, melhorando a hemodinâmica cardiovascular e o terpineol que age de modo a bloquear o influxo de cálcio nos canais e as catequinas agem na musculatura lisa vascular e os alcalóides possuem ação diurética.

No que se refere as interações medicamentosas, Souza et al. (2017) e Lins et al. (2020) estão em concordância que o terpineol presente na planta atua de forma similar medicamentos da classe dos anti-hipertensivos antagonistas do cálcio e agem impedindo a entrada de cálcio nas células e que a utilização de Cardamomo associado com medicamentos dessa classe pode provocar hipotensão porque eleva o efeito anti-hipertensivo e que as catequinas apresentam ação farmacológica similar ao mecanismo de ação dos anti-hipertensivos vasodilatadores diretos e pode potencializar a ação do fármaco quando usados em concomitância com a planta.

\section{Citrus sp.}

A laranja é constituída principalmente por fenóis, taninos, (antocianidinas, flavanonas, flavonóis), xantonas, saponinas, esteroides e triterpenóides a hesperidina e possui atividade hipotensora, bem como possui efeitos $\beta$-bloqueadores, culminando em efeito anti-hipertensivo e inibe a atividade da ECA (Humenhuk et al., 2020).

Silva, Quadros e Neto (2015), Velazquez et al. (2017), trataram acerca do mecanismo de ação e discutiram que a laranja provoca uma redução da pressão arterial. Quanto as interações medicamentosas Silva, Quadros e Neto (2015), acrescentaram que ocorrem interações do tipo sinérgicas com fármacos anti-hipertensivos da classe dos $\beta$-bloqueadores , porque o princípio ativo, hesperidina, presente na planta apresenta ação semelhante de bloqueio dos receptores $\beta$-adrenérgicos, assim como ocorre no fármaco que atuam na redução do débito cardíaco que diminuiu a secreção de renina pelas células justaglomerulares e possuem ação central diminuindo a atividade simpática e que o mecanismo de ação de Citrus $s p$. atua de forma aditiva a atividade do fármaco quando for utilizado em associação.

\section{Passiflora sp.}

Andrade (2018), Santos; Galindo e Queiroz (2020), explicaram que o maracujá é composto principalmente por flavonóides C-glicosilados e saponinas e que o mecanismo de ação reside na redução da pressão arterial devido a ação vasodilatadora de polifenóis como a luteína e seus glicosídeos contidos no extrato, bem como das propriedades antioxidantes dos compostos fenólicos.

Souza et al. (2017) e Costa et al. (2019) citaram que pode ocorrer interações medicamentosas com medicamentos da classe dos vasodilatadores diretos potencializando o efeito farmacológico, visto que, o mecanismo de ação o qual a planta atua é semelhante a essa classe farmacológica.

\section{Lippia alba}

A erva-cidreira é composta por citronelol, citral, mirceno, limoneno e carvona e seu mecanismo de ação envolve o citronelol que é o responsável pela atividade anti-hipertensiva da planta, devido ao efeito direto na musculatura lisa vascular promovendo vasodilatação (Gomes et al.,2016, Barros et al.,2018).

No que diz respeito as interações medicamentosas, o citronelol em virtude de seu mecanismo de ação ser similar aos vasodilatadores diretos que atuam sobre a musculatura da parede vascular, proporcionando relaxamento muscular que resultam em vasodilatação e diminuição da resistência vascular periférica, por isso, a associação entre essa classe de fármacos e a ervacidreira pode causar uma potencialização do efeito do fármaco (Gomes et al.,2016, Costa et al.,2017, Barros et al.,2018). 


\section{Cymbopogon citratus}

O capim-santo é composto por geraniol, neral e mirceno e o mecanismo de ação envolve a redução da resistência vascular, o que pode ser provocada por inibição do influxo de íons de cálcio e ativação de receptores muscarínicos cardíacos que provocam a bradicardia (Velazquez et al., 2017).

Sobre as interações medicamentosas, podem ocorrer interações sinérgicas dos antagonistas dos canais de cálcio, associados ao uso da planta, porque o mecanismo pelo qual os constituintes da planta provocam hipotensão e são similares ao mecanismo de ação dos fármacos das classes da fenilalquilaminas, benzotiazepinas e diidropiridinas. Esses medicamentos ligam-se as subunidades $\alpha 1$ dos canais de cálcio do tipo L e interagem entre si impedindo a abertura dos canais, diminuindo a entrada de cálcio e os efeitos no musculo liso a dilatação arterial generalizada, o que reduz a resistência arteriolar, reduzindo a pressão (Farias, 2016; Velazquez et al., 2017, Souza et al.,2017, Barros et al 2018, Carvalho et al.,2021).

\section{Considerações Finais}

Os estudos ressaltaram que pacientes acometidos por hipertensão arterial sistêmica não devem utilizar plantas medicinais como tratamento coadjuvante sem a orientação profissional, porque podem causar interações com os medicamentos, visto que podem ocasionar quadros de hipotensão bem como potencializar os efeitos colaterais dos medicamentos.

Com base nos estudos discutidos nessa revisão narrativa, as seis plantas com ação anti-hipertensiva são Allium sativum, Alpinia zerumbet, Passiflora sp., Citrus sp., Cymbopogom citratus, Lippia alba. No que concerne as interações relacionadas a utilização de plantas medicinais em concomitância com medicamentos para o tratamento da Hipertensão arterial as mais relatadas foram as farmacodinâmicas com efeitos sinérgico, e as interações farmacocinética, por interferência nas enzimas hepáticas.

Neste estudo foi evidenciado que as classes de medicamentos anti-hipertensivos mais relacionadas com as interações com plantas foram os antagonistas dos canais de cálcio que apresentou interações com o Alho e o Capim-santo, os $\beta$ bloqueadores interagiram com Alho e Laranja, os inibidores da ECA interagiram com Laranja e Alho, os vasodilatadores diretos apresentaram interação com o maracujá e a erva cidreira.

\section{Agradecimentos}

Agradecemos a todos que diretamente e indiretamente contribuíram para a realização e sucesso do artigo.

\section{Referências}

Almeida, G. S., et al. (2016). Conhecimento e uso de plantas medicinais da cultura afro-brasileira pelos moradores da comunidade da Fazenda Velha no munic Ãpio de JequiÃ@)-BA. Revista Veredas da História, 5(2), 27-39.

Almeida, R., et al. (2019). Medidas não farmacológicas em hipertensão arterial. Revista da Faculdade de Ciências Médicas de Sorocaba, 21 (2), 95-97.

Andrade, L. N. (2018). Efeitos biológicos de extratos da Passiflora alata: uma revisão da literatura. Caderno de Graduação-Ciências Biológicas e da SaúdeUNIT-Sergipe, 5(2), 33-65.

Andrade, S. S. de A., et al. (2015). Prevalência de hipertensão arterial autorreferida na população brasileira: análise da Pesquisa Nacional de Saúde, 2013. Epidemiologia e Serviços de Saúde, 24(2), 297-304.

Barbosa-Filho, J. M., et al. (2006). Natural products inhibitors of the angiotensin converting enzyme (ACE): A review between 1980-2000. revista brasileira de farmacognosia, 16(3), 421-446.

Barros, A. L. S., et al. (2018). Utilização de Plantas Medicinais para Ajuda do Controle da Hipertensão. International Journal of Nutrology, 11(01), 77-780.

Campos, M. O., \& Rodrigues Neto, J. F. (2009). Doenças crônicas não transmissíveis: fatores de risco e repercussão na qualidade de vida. Revista Baiana de Saúde Pública, 33(4), 561-581. 
Carneiro, A. L. C., \& Comarella, L. (2016). Principais interações entre plantas medicinais e medicamentos. Revista Saúde e desenvolvimento, 9(5), 4-19

Carvalho, L. O. L., et al. (2021). Atenção farmacêutica no uso de plantas medicinais com ação anti-hipertensiva em idosos. Research, Society and Development, 10(9), 14

Carvalho, M. E. M. de M., Alves, E. L. M., \& Martins, M. do C. de C. (2016). Effect of oral antihypertensive drugs on biochemical dosages in the elderly. Revista da Rede de Enfermagem do Nordeste, 17(6), 11858-865

Carvalho, T. B., et al. (2015). Papel dos idosos no contexto do uso de plantas medicinais: contribuições à medicina tradicional. Ensaios e Ciência: Ciências Biológicas, Agrárias e da Saúde, 19(1), 38-41

Castro, K. N. de C., et al. (2018). Composição química e eficácia do óleo essencial e do extrato etanólico de Alpinia zerumbet sobre Staphylococcus aureus. Arquivos do Instituto Biológico, 83, 1-7

Chequer, G. et al. (2018). Segunda opinião formativa: no tratamento da hipertensão, como realizar a escolha inicial e a progressão dos medicamentos antihipertensivos? Revista Brasileira de Hipertensão, 29(10), 47-51

Costa, A. R. F. C., et al. (2018). Uso de plantas medicinais por idosos portadores de hipertensão arterial. Revista de Ciências da Saúde Nova Esperança, 17(1), $16-28$

Costa, P. S., et al. (2017). Atividade antimicrobiana e potencial terapêutico do gênero Lippia sensu lato (Verbenaceae). Hoehnea, 44, 158-171

Cruz, I. C. F. (2015). What is the best anti-hypertensive for Black Brazilian Population? Journal of Specialized Nursing Care, 7(2), 1-17

Cunha, G. H. et al. (2016). Estudo comparativo do efeito anti-hipertensivo das frações hexânixa, clorofórmica e metanólica do óleo essencial da Alpinia zerumbet. Revista Brasileira de Plantas Medicinais, 18, 113-124

Cupertino, L. H., et al. (2016). Medicamentos anti-hipertensivos dispensados em uma unidade básica de saúde no município de Ipatinga-MG. ÚNICA Cadernos Acadêmicos, 3(1), 1-13

Dallacosta, F. M. (2015). Perfil de Hipertensos Cadastrados no Programa Hiperdia de uma Unidade Básica de Saúde. Unoesc \& Ciência - ACBS, Joaçaba. $1(1), 45-52$

Dias, E. C. M., et al. (2017). Uso de fitoterápicos e potenciais riscos de interações medicamentosas: reflexões para prática segura. Revista Baiana de Saúde Pública, 41(2), 297-303

Dias, E. G., et al. (2016). Lifestyle and hindering factors in controlling hypertension/Estilo de vida e fatores dificultadores no controle da hipertensão/Estilo de vida y factores que complica el control de la hipertensión. Revista de Enfermagem da UFPI, 4(3), 24-9

Elliott, W. J., \& Ram, C. V. S. (2011). Calcium channel blockers. Journal of Clinical Hypertension, 13(9), 687-689

Farias, D. (2016). Uso de plantas medicinais e fitoterápicos como forma complementar no controle da hipertensão arterial. Journal of Biology \& Pharmacy and Agricultural Management, 12(3), 19-27

Feitosa, A. D. M, et al. (2020). Tratamento Medicamentoso da Hipertensão: Do Trio de Ouro ao Octeto. Arquivos Brasileiros de Cardiologia, 115, 270-272

Freitas, J. G. A., Nielson, S. E. de O., \& Porto, C. C. (2015). Adesão ao tratamento farmacológico em idosos hipertensos: uma revisão integrativa da literatura. Rev Soc Bras Clin Med, 13(1), 75-84

Gil, A. C. (2006). Como elaborar projetos de pesquisa. (4a ed.), Atlas

Gomes, J. V. D., et al. Triagem fitoquímica e avaliação das atividades trombolítica e citotóxica de cecropia hololeuca miq.(urticaceae), lippia alba (mill.) Ne br. Ex p. Wilson (verbenaceae) e zanthoxylum rhoifolium lam (RUTACEAE). Infarma-Ciências Farmacêuticas, 28(1), 10-15

Hasenclever, L. et al. (2017). A indústria de fitoterápicos brasileira: desafios e oportunidades. Ciência \& Saúde Coletiva, 22, 2559-2569

Humenhuk, T., Leite, D. R. B., \& Fritsch, M. (2020). Conhecimento popular sobre plantas medicinais utilizadas no município de Mafra, SC, Brasil. Saúde e meio ambiente: revista interdisciplinar, $9,27-42$

Issa, A. F. C., et al. (2021). Diretrizes Brasileiras de Hipertensão Arterial-2020. Arq Bras Cardiol, 116(3), 516-658, 2021.

Lee, Y-M., et al. (2016). Effect of a brown rice based vegan diet and conventional diabetic diet on glycemic control of patients with type 2 diabetes: a 12 -week randomized clinical trial. PloS one, 11(6), 1-14

Lima, A. R. N. et al. (2018). Efeitos do Alho (Allium Sativum L.) Sobre a Hipertensão Arterial Sistêmica. International Journal of Nutrology, 11(1), 220-230

Lima, M. R., et al. (2020). Efeito terapêutico do allium sativum 1. N no controle da hipertensão arterial. Revista de Atenção à Saúde. 18(65), 1-14

Lins, S. R. et al. (2020). Aplicações terapêuticas da Alpinia Zerumbet (colônia) baseado na medicina tradicional: uma revisão narrativa (2010-2020). Brazilian Journal of Development, 6(1)1,84222-84242

Lucena, J. A. S., \& Guedes, J. P. (2020). Uso de fitoterápicos na prevenção e no tratamento da hipertensão arterial sistêmica. Revista Brasileira de Educação e Saúde, 10(1), 15-22

Machado, J. C., et al. (2016). Adesão ao tratamento não farmacológico: análise do impacto de três estratégias de educação em saúde e nutrição em portadores de hipertensão arterial. Revista de Nutrição, 29, 11-22 
Magnabosco, P., et al. (2015). Análise comparativa da não adesão ao tratamento medicamentoso da hipertensão arterial sistêmica em população urbana e rural. Revista Latino-Americana de Enfermagem, 23(1), 20-27.

Malachias, M. V. B. (2016). et al. $7^{\text {a }}$ Diretriz Brasileira de Hipertensão Arterial: capítulo 2-diagnóstico e classificação. Arquivos Brasileiros de Cardiologia, 107(3), 7-13.

Mascarenhas, W. C., et al. (2016). Prevalência de pré-hipertensão e de hipertensão arterial e sua associação com variáveis antropométricas e estado nutricional de pré-escolares. Revista Brasileira de Hipertensão, 23(2), 47-51.

Minayo, M. C. de S., \& Souza, C. (2010). O desafio do conhecimento: pesquisa qualitativa em saúde. (8a ed.) Hucitec, 201-219.

Monteiro, J. R. G. C. (2017). Protocolo clínico de avaliação e acompanhamento dos pacientes hipertensos. Revista Brasileira de Hipertensão, 11(2), 20-30

Nascimento, J. S., Gomes, B., Lima Sardinha, A. H. (2011). Fatores de risco modificáveis para as doenças cardiovasculares em mulheres com hipertensão arterial. Northeast Network Nursing Journal, 12(4), 709-715

Neto, C., et al. (2017). Clonidine versus captopril for severe postpartum hypertension: a randomized controlled trial. PloS one, 12(1), 13-24

Nobre, F., et al. (2013). Hipertensão arterial sistêmica primária. Medicina (Ribeirao Preto. Online), 46(3), 256-272

Nogueira, D., et al. (2014). Reconhecimento, tratamento e controle da hipertensão arterial: Estudo Pró-Saúde, Brasil. Revista Panamericana de Salud Publica, 27, 103-109

Oigman, W., Neves, F. M., \& Gismondi, R. A. O. C. (2015). Hipertensão arterial sistêmica. Arterial hypertension. Rev Bras Med, 72(1/2), 5-17

Oliveira, C. J., \& Araujo, T. L. (2017). Plantas medicinais: usos e crenças de idosos portadores de hipertensão arterial. Revista Eletrônica de Enfermagem, $9(1), 1-13$

Oliveira, T. L., et al. (2013). Eficácia da educação em saúde no tratamento não medicamentoso da hipertensão arterial. Acta Paulista de Enfermagem, 26(2), $179-184$

Pereira, J. B. A. et al. (2015). O papel terapêutico do Programa Farmácia Viva e das plantas medicinais. Revista Brasileira de Plantas Medicinais, 17, 550-561

Peres, H. A., \&Castro, S. H. R. (2015). Tratamento farmacológico e interações medicamentosas em pacientes com aneurisma da aorta abdominal. InfarmaCiências Farmacêuticas, 27(2), 135-141

Pontes, S. M., et al. (2012). Utilização de plantas medicinais potencialmente nocivas durante a gestação. Comunicação em ciêNcias da Saúde, $23(4), 305-311$.

Porto, D., et al. (2015). Uso de medicamentos por pessoas idosas na comunidade: proposta de ação de enfermagem. Revista Brasileira de Enfermagem, 66(5), 703-708.

Reis Filho, R. A. R. J. (2015). Reverse Cardiac Remodeling: A Marker of Better Prognosis in Heart Failure. Arq. Bras. Cardiol., 104(6), 502-506.

Ribas, S. A., et al. (2014). Fatores de risco cardiovascular e fatores associados em escolares do Município de Belém, Pará, Brasil. Cadernos de Saúde Pública, $30(3), 577-586$

Santos, A. P. M., Galindo, A. S., \& Queiroz, E. (2020). Propriedades neuropsicofarmacológicas, compostos quimicamente ativos e uso medicinal da passiflora incarnata. Brazilian Journal of Development, 6(12),94823-94836.

Santos, S. L. F., et al. (2016). Uso de plantas medicinais nos serviços do sistema único de saúde: uma revisão narrativa. Revista Saúde \& Ciência Online, 5(3), 63-80.

Serrate Mengue, Sotero et al. Acesso e uso de medicamentos para hipertensão arterial no Brasil. Revista de Saúde Pública, 50(2), 1-9, 2016.

Silva, L. F. da R. S. et al. (2015). Fatores associados à adesão ao tratamento anti-hipertensivo por idosos na atenção primária. Revista de Ciências Farmacêuticas Básica e Aplicada, 35(2), 271-278

Silva, L. E., Quadros, D. A., \& Neto, A. J. M. (2015). Estudo etnobotânico e etnofarmacológico de plantas medicinais utilizadas na região de Matinhos-PR. Ciência e Natura, 37(2), 266-276.

Simão, R. R., et al. (2016). Hipertensão Arterial Sistêmica (HAS). Revista de Medicina, 95(1), 37-38.

Souza, J. B. P. et al. (2017). Interações planta medicinal x medicamento convencional no tratamento da hipertensão arterial. Infarma Ciências Farmacêuticas, 29(2), 90-9.

Suchard, M. A. et al. (2020). Comparação abrangente de eficácia e segurança das classes de medicamentos anti-hipertensivos de primeira linha: uma análise sistemática, multinacional e em grande escala. Rev Bras Hipertens, 27(4), 141-152

Vejakama, P. et al. (2012). Reno-protective effects of renin-angiotensin system blockade in type 2 diabetic patients: A systematic review and network metaanalysis. Revista de Diabetologia, 55(3), 566-578.

Velazquez, M. del R. H., Palmero, O. R., \& Cortiñas, L. T. (2017). Plantas popularmente antihipertensivas en Jipijapa, Manabí. Mito y realidad. Revista Amazónica Ciencia y Tecnología, 6(2), 113-125

Vongpatanasin, W. et al. (2011). Central Sympatholytic Drugs. The Journal of Clinical Hypertension, 13(9), 658-661.

Yugar-Toledo, J. C., et al. (2020). Posicionamento Brasileiro sobre Hipertensão Arterial Resistente-2020. Arquivos Brasileiros de Cardiologia, 114, 576-596.

Weber, D., Oliveira, K. R. de, \& Colet, C. de F. (2014). Adesão ao tratamento medicamentoso e não medicamentoso de hipertensos em Unidade Básica de Saúde. Rev Bras Hipertens, 21(2), 114-121. (2014). 